\title{
Gradhiva
}

GRADHIV

Revue d'anthropologie et d'histoire des arts

$1 \mid 2005$

Haîti et l'anthropologie

\section{Rendez-vous manqué avec le vodou}

Jean Jamin

\section{OpenEdition}

Journals

Édition électronique

URL : http://journals.openedition.org/gradhiva/375

DOI : $10.4000 /$ gradhiva.375

ISSN : $1760-849 x$

\section{Éditeur}

Musée du quai Branly Jacques Chirac

\section{Édition imprimée}

Date de publication : 1 mai 2005

Pagination : 225-231

ISBN : 2-915133-08-5

ISSN : 0764-8928

\section{Référence électronique}

Jean Jamin, «Rendez-vous manqué avec le vodou », Gradhiva [En ligne], 1 | 2005, mis en ligne le 10 décembre 2008, consulté le 02 mai 2019. URL : http://journals.openedition.org/gradhiva/375 ; DOI 10.4000/gradhiva.375

Ce document a été généré automatiquement le 2 mai 2019.

(c) musée du quai Branly 


\title{
Rendez-vous manqué avec le vodou
}

\author{
Jean Jamin
}

Le texte de Michel Leiris que nous reproduisons ici - extrait d'un de ses carnets de route tenus lors d'une mission ethnographique en Martinique, Guadeloupe et Haït, du 26 juillet au 12 novembre $1948^{1}$ - a fait l'objet d'une première publication en décembre 1951, sous le titre « Sacrifice d'un taureau chez le houngan Jo Pierre-Gilles », dans un numéro spécial de la revue Présence africaine ( $\left.n^{\circ} 12\right)$ consacré à « Haïti, poètes noirs », et dont l'ensemble fut supervisé par Alfred Métraux (pp. 22-36 de ladite livraison). Il a été repris en 1981, sous la forme d'une plaquette de 32 pages et avec le même titre, par un petit éditeur d'Amiens, Le

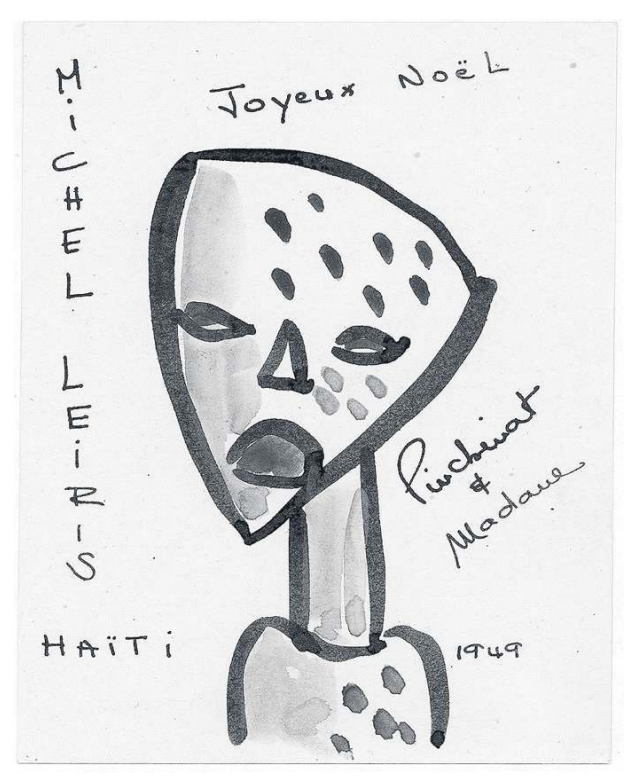
Nyctalope (tirage limité à 549 exemplaires), sans les illustrations (quatre photographies dont deux d'Alfred Métraux et deux de Pierre Verger, figurent dans l'original) ; l'exorde fut abrégé et renvoyé en note par Leiris, juste avant le colophon (dans cette présente édition, nous l'avons rétabli et replacé en tête de l'article).

2 Ce sera, hormis quelques paragraphes de la longue introduction au numéro thématique des Temps Modernes que Leiris a coordonné en 1950 (" Martinique, Guadeloupe, Haïti », n 52, pp. 1345-1368) et la note sur l'« usage de chromolithographies catholiques par les vodouïsants $»^{2}$, l'un des rares articles qu'il a publiés de son vivant sur l'ethnographie haïtienne et, en particulier - lui qui ne cachait pas son engouement pour ce que Métraux qualifiait de « comédie rituelle », - sur le vodou ${ }^{3}$. 
Il faut dire que la réception de son article de Présence africaine ne devait guère l'encourager dans cette voie, non pas tant à cause du contenu (l'acuité du regard de Leiris, l'authenticité de l'observation, la véridicité des faits et épisodes rituels rapportés ne furent jamais mis en doute) mais de la forme qu'il lui donna: divulgation de fragments d'un carnet intime, simplement accompagnés de notes additionnelles et explicatives écrites pour la circonstance et composées en bas de page.

Marché au bord de la Gosseline

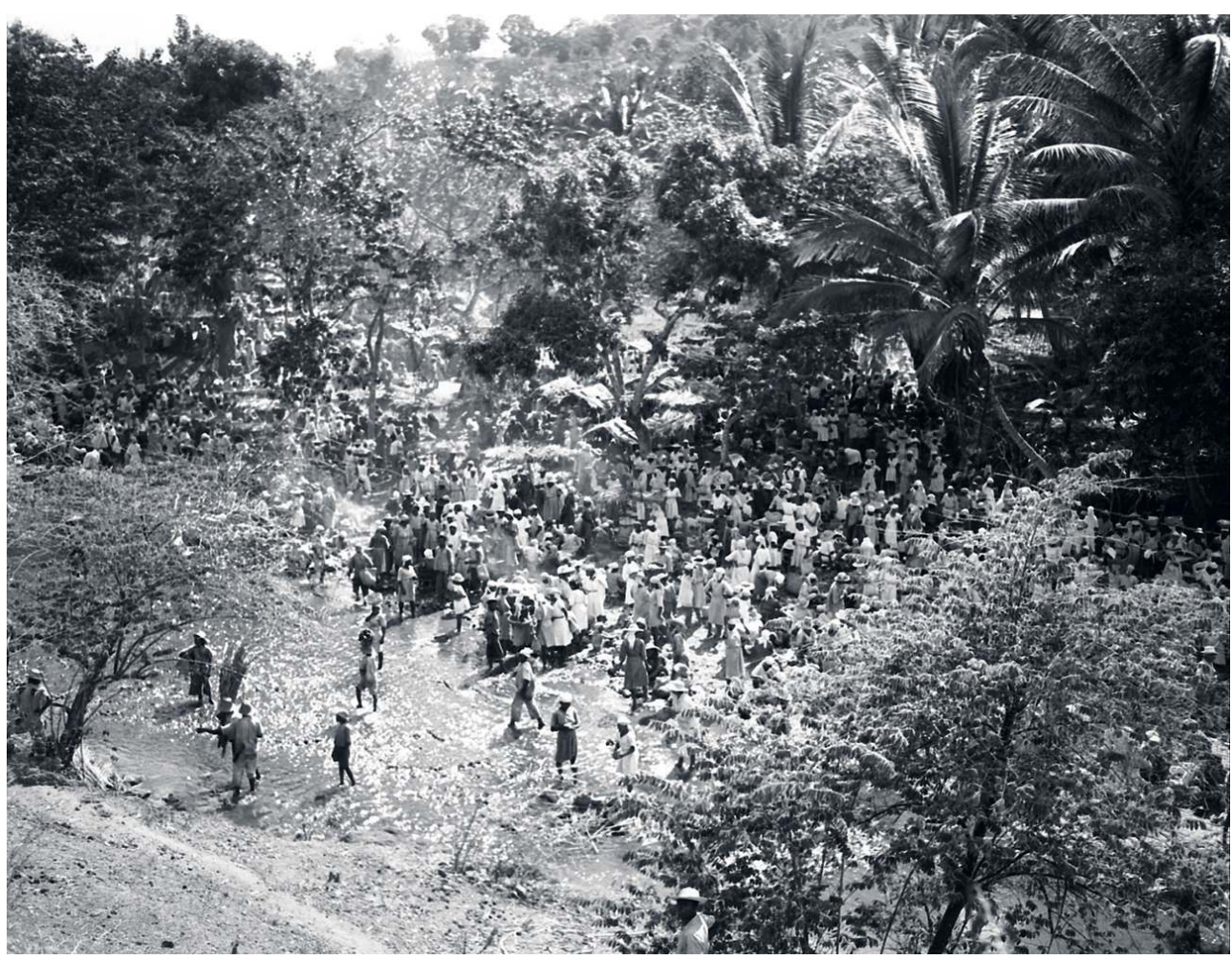

(c) UNESCO

4 Odette Mennesson-Rigaud, la principale informatrice, guide et interprète de Leiris ${ }^{4}$ - elle l'accompagna dans presque toutes les séances de vodou auxquelles il lui fut offert d'assister - n'hésita pas à le tancer. Au-delà du cas particulier de ce texte dont elle juge la publication inopportune et déloyale aussi bien envers elle qu'envers ceux qui le reçurent, Odette Mennesson-Rigaud, en toute connaissance de cause semble-t-il, pose là un problème clé de l'enquête ethnographique, longtemps négligé, et qui, de toute évidence, devient singulièrement aigu dès lors que l'enquête s'exerce sur des terrains en vérité proches culturellement (ne serait-ce que par la langue) et donne lieu à ce que je proposerai d'appeler une lecture participante de ses résultats - c'est-à-dire un problème d'exposition, d'expression, d'élaboration et de diffusion des matériaux recueillis. En plus des questionnements d'ordre éthique et épistémologique qu'engendrent les modalités de la restitution des informations en ethnologie, celles-ci peuvent avoir des incidences politiques locales surtout lorsque les circonstances mettent nommément en cause l'objet desdites informations : c'est le cas du vodou (et de ses adeptes), qui, sous prétexte d'une modernisation accélérée de la société haïtienne suivant un modèle libéral, est alors frappé d'interdiction (dès 1939, le président Élie Lescot soutenu par l'Église catholique avait lancé une campagne anti-superstitieuse ${ }^{5}$ ). Dans une lettre qu'elle adresse à Leiris le 
16 février $1952^{6}$, Odette Mennesson-Rigaud écrit en effet (il importe de citer le passage de cette lettre en entier) :

«Mon cher Leiris ${ }^{7}$

[...] Je vous avoue que j'ai été plus encore peinée qu'indignée en lisant votre article paru dans le numéro de Présence africaine. Non seulement vous désignez nommément le houngan qui vous avait reçu - à votre satisfaction me semble-t-il mais vous ajoutez des commentaires qui vont certainement le blesser infiniment. Non seulement vous avez mal agi envers lui (permettez-moi de vous le dire) mais vous me mettez dans une position plus que désagréable vis-à-vis de lui. Plus d'une fois vous citez mon nom pour me faire dire les choses qui peuvent lui être le plus nuisible. Pourquoi tant de méchanceté ? Avez-vous oublié le caractère de notre milieu? Le pauvre houngan paiera bien cher l'honneur de vous avoir reçu, et accepté librement durant son service. Vous ne pouvez pas prétendre que vous ignoriez que le vodou est interdit en Haïti, et que mille entraves viennent gêner ces pauvres gens. Ils sont toujours à la merci des autorités qui ferment souvent les yeux, mais les ouvrent brutalement lorsqu'une preuve est apportée par un "Blanc" que le vodou existe réellement en Haïti, non pas comme réunion de danse, mais comme services rituels. Impossible cette fois de prétendre que le "Blanc" a imaginé la scène rapportée. Trop de noms et de précisions viennent à l'appui du réquisitoire. Ainsi que je l'ai écrit au docteur Métraux - ayant parlé de vous il m'a paru correct de vous faire tenir la copie ci-jointe ${ }^{8}$ - on m'a déjà fait part $d u$ mécontentement qui a suivi la divulgation de ces notes. Je crois qu'après cela il m'est impossible de retourner du côté de la Croix des Missions. On m'a même avertie d'avoir à prendre certaines précautions. Croyez-vous que cela m'enchante? Il est bien agréable, n'est-ce pas, d'être l'ethnographe qui ne fait que passer... Si vous pensez que j'exagère un peu, vous n'avez qu'à vous rappeler la rancune tenace que l'on a gardée à Paul Reboux pour ses notes de voyage parues sous le titre Blancs et Noirs.»9

5 On ignore ce que furent les réponses de Leiris et de Métraux, si ce n'est par une lettre d'Odette Mennesson-Rigaud à Leiris, en date du 6 mars 1952, qui se déclare rassurée - elle renoue avec «Mon cher Ami »- même si l'un des arguments apparemment utilisé par Leiris, et qu'elle cite, à savoir que Présence africaine ne s'adresse qu'à un "étroit milieu intellectuel s'intéressant à l'Afrique ", ne la convainc pas. Elle s'emploie non sans vigueur et rigueur à le démonter, observant que ce sont justement de tels gens qui, en Haïti, sont « le plus touchés par le vodou », et elle ajoute :

«Beaucoup d'étudiants, troublés par les traditions ancestrales ou familiales, viennent chercher une explication à l'Institut d'ethnologie ou dans les livres traitant de cette question. Ce sont eux aussi, ces étudiants, qui, fréquentant des houmfors [temples vodou], seront amusés de reconnaître tel ou tel [...]. Maintenant le livre [le numéro de Présence africaine] circule sous les péristyles, bien et mal lu ! Et personne ne fera assez attention aux noms des dirigeants de cette revue pour se dire que ce ne sont pas des Blancs... $»^{10}$

6 Toujours est-il que, malgré les craintes et ressentiments d'Odette Mennesson-Rigaud visà-vis de cette parution, l'affaire sembla en rester là. Leiris et elle continuèrent de s'écrire, irrégulièrement il est vrai (la correspondance s'interrompt entre décembre 1953 et septembre 1958) ${ }^{11}$, et ce jusqu'au début des années 1960, sans qu'apparussent dans les missives de celle-ci d'autres ou nouvelles allusions aux effets éventuellement dévastateurs des « révélations » qu'aurait faites celui-là dans son article.

7 Qu'Odette Mennesson-Rigaud ait surestimé les conséquences de cette publication, cela est probable, d'autant plus que son mari, Milo Rigaud, est alors emprisonné par le pouvoir haïtien $^{12}$ et qu'elle se sent donc prise dans le collimateur. Mais sa réaction vive et à vif 
vient stigmatiser, comme on l'a suggéré plus haut, la position et la posture de l'ethnographe qui, animé des meilleures intentions, soucieux de se placer sur le même plan que ses interlocuteurs, habité de convictions humanistes, taraudé par la question du colonialisme $^{13}$ ou du néocolonialisme ${ }^{14}$, ne saurait seulement se préoccuper des conditions souvent bien peu sereines et équitables d'obtention de ses informations ni des relations - généralement aussi peu sereines et équitables - qu'il est amené à nouer avec ceux qui les lui procurent (Leiris, certes, par sa courtoisie et sa discrétion, s'est employé dans le cas présent à les lisser, comme d'ailleurs ne manque pas de lui signifier Odette Mennesson-Rigaut $\left.{ }^{15}\right)$. D'autres conditions, notamment celles qui concernent la restitution des informations, sous quelque forme que ce soit, à qui que ce soit et où que ce soit, sont donc tout aussi sinon plus importantes ${ }^{16}$. C'est ce que, non sans malice, rappelle aussi Odette Mennesson-Rigaud à Leiris, lui faisant en quelque sorte une leçon d'ethnographie pratique lorsqu'elle lui écrit (lettre du 6 mars 1952):

«Ce qui me paraissait inexplicable, c'est que le numéro ayant été fait sous la supervision du Professeur Métraux et ayant souvent attiré son attention sur la nécessité de déguiser les noms de lieux et de personnes (déjà, depuis mon étude dans Primitive $\mathrm{Man}^{17}$ ), il aurait certainement dû vous faire remarquer l'importance de la chose. »

8 Même traversée et chahutée par les esprits du vodou, Haïti n'est pas, n'est plus une Afrique fantôme... Leiris, qui venait ainsi d'opérer à la manière de son journal tenu lors de la Mission Dakar-Djibouti, c'est-à-dire publié tel quel, sans ajouts, retouches, biffures ou maquillages, l'apprenait à ses dépens, comme du reste il l'avait appris après la parution de L'Afrique fantôme en 1934, à cette différence près - elle est de taille - que les réticences et remontrances étaient venues de ses « maîtres blancs » (en l'occurrence Marcel Mauss, Marcel Griaule et Paul Rivet), non point de ses informateurs dogon ou amhara ${ }^{18}$.

En éprouva-t-il de la honte? En nourrit-il un sentiment de culpabilité ? En eut-il du remords? Rien dans ses écrits de l'époque, qu'ils soient autobiographiques, épistolaires ou ethnologiques, ne le laisse supposer. Ce qui ne signifie nullement que la question ne se pose pas. Son attitude, dès lors faite d'évitement et de silence, incite à penser que cet impair vis-à-vis des vodouïsants (Leiris demeure un " gaffeur » impénitent ${ }^{19}$ ) ne fut pas sans incidence tant sur le plan méthodologique, voire épistémologique, que sur le plan technique, scripturaire: bien qu'il ne se départît jamais de cette habitude de tenir un journal pendant ses voyages - qui doublait ou remplaçait momentanément celui que, depuis 1922, il continuait d'écrire à domicile -, Leiris ne publiera plus de son vivant ne serait-ce qu'un court extrait de ses notes prises sur le vif. 


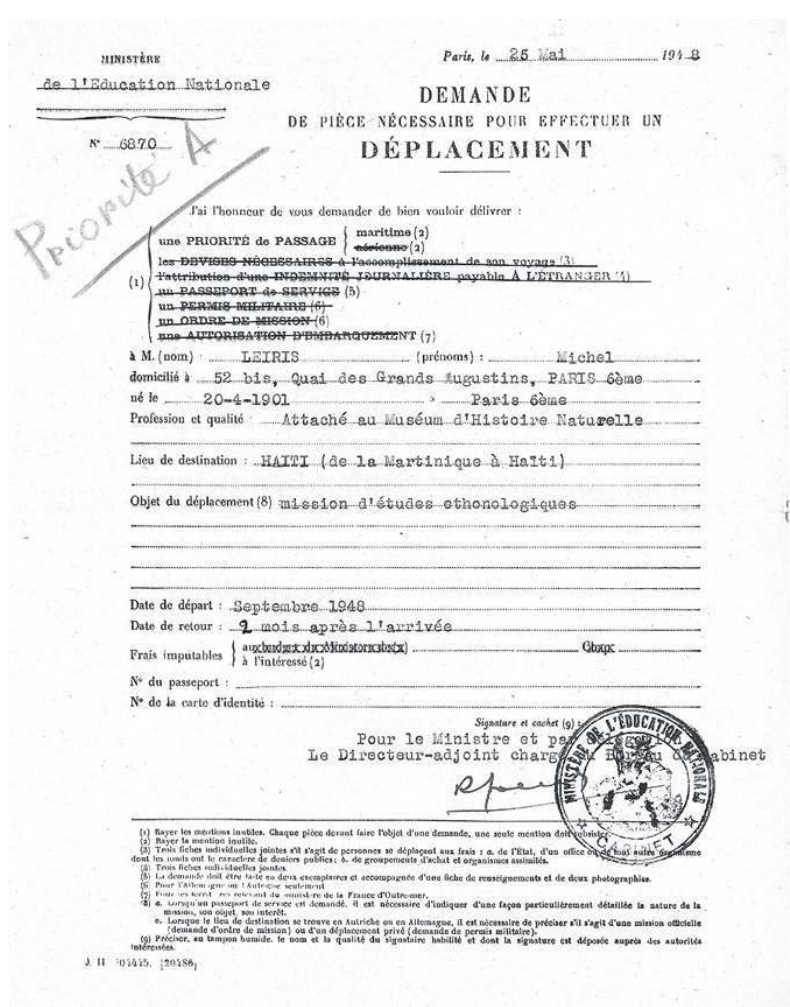

(c) Laboratoire d'Anthropologie sociale, droits réservés

10 C'est non seulement la notion de document, sa brutalité circonstancielle, sa prétendue sédimentation sémique, sa supposée « fraîcheur » qui en prennent un coup ${ }^{20}$ - il n'existe pas de données compactées pas plus qu'il n'y a de faits monolithiques ou d'objets univoques, ou encore de documents censés cristalliser ou refléter un rite, une culture, une société -, mais celle d'observation ethnographique, laquelle ne peut être «participante» qu'à condition de tenir et rendre compte des interactions qu'elle ne saurait manquer de provoquer, fût-ce dans l'après-coup, après l'enquête. Comme le signalait Jean Bazin ${ }^{21}$, ce ne sont pas des cultures, des rituels, des symboles, des comportements - des choses sociales - que l'ethnographe observe, mais tout d'abord des situations et des actions humaines, c'est-à-dire des configurations particulières et temporaires dont il s'agit de décrire et de comprendre les règles du jeu et d'en énoncer ainsi «le champ des "coups" possibles ou plausibles» ${ }^{22}$, y compris, pourrions-nous ajouter en filant la métaphore, celui de la «donne » et de la «main», autrement dit: la distribution et la répartition du ou des textes ethnographiques auxquelles ces règles $d u$ jeu vont donner lieu. De retour chez soi, l'ethnographe n'est pas pour autant quitte d'avoir été ailleurs et chez d'autres gens : d'eux tout ne peut être dit ni écrit comme ne peut l'être - et Leiris allait en faire une autre cruelle expérience avec son entreprise autobiographique ${ }^{23}$ - tout de soi.

11 Il ne retournera jamais en Haïti, bien qu'il eût tout loisir de le faire lors de sa seconde mission ethnographique aux Antilles que la parution du numéro de Présence africaine précède de quelques mois (Leiris y séjourne du 21 mars au 12 juillet 1952). Il ne tiendra pas au courant Odette Mennesson-Rigaut de ce second voyage; elle ne l'apprendra que par Métraux, en septembre 1953. Dans l'ouvrage qu'il écrit au retour de cette mission et 
qu'il limite aux seules Antilles françaises ${ }^{24}$, les problèmes rituels et religieux sont à peine esquissés et pas du tout traités. Tout se passe comme si Leiris avait voulu tirer un trait sur sa précédente expérience même si - mais cette fois à titre personnel - il lui arrive de l'évoquer dans Fourbis. Plus particulièrement, tout se passe comme s'il avait voulu effacer le vodou haïtien de sa mémoire ethnologique, alors qu'un de ses penchants pour ainsi dire naturels l'avait amené à placer les phénomènes de possession au centre de ses préoccupations anthropologiques. Il serait sans doute excessif de croire que la réaction d'Odette Mennesson-Rigaud à la publication de son texte ait pu occasionner une telle mise entre parenthèses (on l'a vu, dès mars 1952, les relations sont redevenues normales entre eux et il n'y a donc aucune raison de penser qu'il puisse se sentir « grillé » en Haïti). Sans négliger cependant cet embarras dans lequel Leiris se trouve probablement plongé, d'autres facteurs entrent en ligne de compte.

D'une part, ses liens d'amitiés avec Alfred Métraux qui, engagé depuis plus longtemps que lui dans l'ethnographie haïtienne ${ }^{25}$, devait aux yeux de Leiris conserver la primeur sur l'étude du vodou: ce sera en fait le cas avec la publication en 1958 de son importante monographie sur ce culte (op. cit., 1968), dont il convient de noter qu'elle paraîtra dans la collection « L'espèce humaine » que, depuis 1948, Leiris dirige aux éditions Gallimard ${ }^{26}$.

D'autre part, la réflexion de Leiris sur les phénomènes de possession a quelque peu évolué : elle semble se focaliser sur la transe et sur la dimension esthétique que désormais il s'attache à faire prévaloir et à analyser. Sont mis en avant, non plus l'érotisme sombre et la religiosité sauvage du rite - la " religion du sang »-, non plus les troubles ou écarts de la personnalité, mais le « caractère institutionnel » et l'« allure spectaculaire », c'est-àdire théâtrale, des séances de possession.

«Il serait erroné, écrit-il, de [les] regarder comme exclusivement médico-magiques et, plus encore, [de les] envisager sous l'angle de la psychopathologie. En effet, on constate, d'une part, que les maux attribués au mécontentement des zar et qu'on s'efforce de soigner sont, dans la plupart des cas, des maux d'ordre physique, non d'ordre psychique, constatation qui vaut aussi pour le vaudou. Et l'on observe, d'autre part, que la possession représente, plutôt qu'un mal dont il s'agirait de délivrer le patient, une technique traditionnelle qui permettra censément de le guérir, en apaisant au moyen d'offrandes les zar interrogés par son truchement, et de lui assigner finalement un génie protecteur définissant sa place dans le groupe. Ainsi, l'ensemble du processus apparait comme une sorte d'initiation dont le point de départ aura été la maladie ou le dommage quelconque qu'il convenait de réparer. Dans le cas du vaudou comme dans celui du zar, les crises de possession tendent à constituer une sorte de théâtre vécu, mettant en jeu des personnages mythiques mais conçus comme réels et incarnés, plutôt que joués, par ceux ou celles qui temporairement les endossent. ${ }^{27}$

Sans doute est-ce sur ce point - la théâtralisation de la possession, alors conçue, pour reprendre une de ses expressions, comme un « vestiaire de personnalités »- que Leiris se sépare de ses premières observations et interprétations. Lors de l'édition critique de Miroir de l'Afrique, Jacques Mercier et moi nous avions observé que de L'Afrique fantôme (1934) à La Possession et ses aspects théâtraux... (1958), Leiris semblait être passé d'une conception sacrificielle de la possession - insistant sur la «circulation du mal», les transferts de maladies et de "génies", et sur le sacrifice comme moteur de la relation avec le sacré ${ }^{28}$ - à une conception théâtrale de celle-ci, d'où la dimension proprement dite sacrificielle, cependant fondamentale dans l'efficacité symbolique des rites de possession, était sinon exclue du moins considérablement atténuée dans les descriptions et analyses qu'il en proposa. Les influences de Jean-Paul Sartre (avec la notion de «mauvaise foi ») et 
d'Alfred Métraux pour qui la possession pouvait se concevoir comme une sorte de " comédie rituelle $»^{29}$, ont sans doute été pour beaucoup dans ce changement d'approche. Est-ce donc cette question du sacrifice à laquelle pourtant il accorda toute son attention, ne serait-ce que par cette publication dans Présence africaine, qui l'embarrassait, alors qu'il pensait l'avoir dépassée ? Ce n'est que bien plus tard, en tout cas, dans le seul article qu'il ait donné au Dictionnaire des civilisations africaines ${ }^{30}$, à l'entrée zar, qu'il tente, comme le note Jacques Mercier ${ }^{31}$, d'harmoniser la logique sacrificielle et la théâtralité des cultes de possession.

\section{Dessin d'Odette Mennesson-Rigaud}

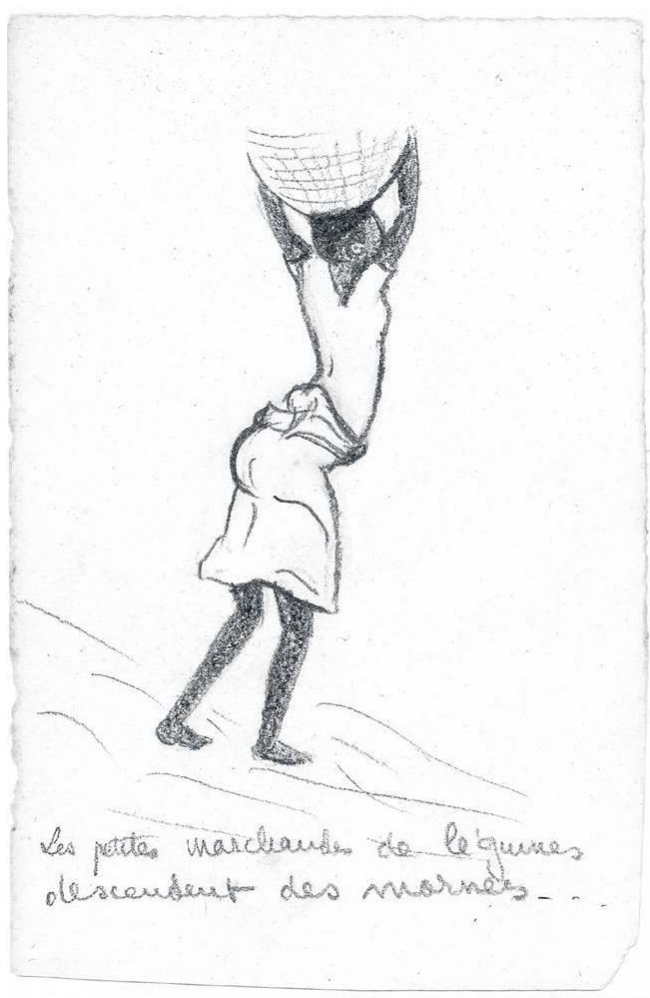

(c) Laboratoire d'Anthropologie sociale, droits réservés

Il reste que Leiris, malgré son désir de vouloir " en être », d'entrer dans le jeu rituel, de se situer de plain-pied avec ses interlocuteurs vodouïsants - comme il l'avait assouvi, en 1932, chez les adeptes du zar éthiopien -, ne put évoluer, faute de temps mais faute aussi de se donner du temps, qu'à la surface des choses - observateur certes attentif et minutieux (comme en témoignent les fragments reproduits ci-dessous), mais observateur pressé qui s'accommode de prises de vue qu'il ne parviendra pas à convertir en prises de date : l'étude comparative que, nourri de sa propre expérience et des notes que lui confia Métraux, il escomptait produire ne se réalisera pas. Et ce ne sont certes pas les quelques commentaires de bas de page qu'il rédige pour La Possession et ses aspects théâtraux... et où il les mentionne - ouvrage paru en même temps que Le Vaudou haïtien de Métraux et, d'une certaine manière, concurremnent avec lui -, qui peuvent en tenir lieu.

En ce sens, le sacrifice du taureau de Seyfou Tchenger - article qui, écrit en 1933 à partir de son journal intime, inaugure ses études sur la possession par les génies $z a r^{32}-$ ne se prolonge ni ne se résout dans le sacrifice du taureau chez Jo Pierre-Gilles, même si les deux textes sont d'abord mis à l'enseigne de l'art et de la poésie : l'un publié dans une 
revue artistique et littéraire, Minotaure, l'autre dans un numéro spécial d'une revue culturelle, Présence africaine, consacrée aux « poètes noirs » haïtiens. Mais n'est-ce pas, au fond, ce que recherchait avant tout Leiris : disons la poétique plutôt que l'ethnographique de la possession qui amène l'homme, d'où qu'il soit, où qu'il naisse, à ne pas se contenter d'être ce qu'il est et à s'inventer d'autres mondes, ne fût-ce qu'en jouant avec l'ombre de la corne d'un taureau?

\section{NOTES}

1. Chargé de mission auprès du ministère des Affaires étrangères (service des relations culturelles), bénéficiaire d'une bourse accordée à l'occasion du centenaire de la Révolution de 1848 et de l'abolition de l'esclavage dans les colonies françaises, encouragé par Alfred Métraux qu'il retrouve à Haïti ainsi que par Aimé Césaire dont il fait alors la connaissance et deviendra un ami proche, Michel Leiris va entreprendre, du 24 septembre au 26 octobre 1948, une enquête intensive sur le vodou haïtien, espérant en tirer des éléments de comparaison avec le culte de possession par les génies zar qu'il avait observé en Éthiopie au cours de la Mission Dakar-Djibouti de 1931-1933 (cf. Michel Leiris, L'Afrique fantôme, in Miroir de L'Afrique, Paris, Gallimard, 1996). La série des quatre carnets de route de Leiris aux Antilles en 1948 (toujours inédite) est conservée à la Bibliothèque littéraire Jacques-Doucet.

2. In Mémoires de l'Institut français d'Afrique noire, 1953, n² 27, pp. 201-207, repris dans Brisées, Paris, Gallimard, 1992, pp. 176-189.

3. Dans son carnet haïtien ( $\left.n^{\circ} 3\right)$, à la date du 19 octobre 1948, Leiris note : «Avec [Alfred] Métraux et Jeanne Sylvain au Kalmar's Café [Port-au-Prince], à bavarder vaudou et ethnographie. J'explique comment le théâtre occidental contemporain me paraît d'une pauvreté désolante à côté des manifestations vaudou (comparables à ce que fut la tragédie grecque), manifestations dans lesquelles le spectateur est entraîné dans l'action et dans lesquelles le spectateur se sent même, parfois, menacé par cette action. Déjeuner avec Métraux où je parle toujours vaudou: comment l'esprit possesseur intervient à la fois comme explication de l'état et comme formulation et stylisation de cet état ;.../...

4. Française d'origine, devenue haïtienne par son mariage, Odette Mennesson-Rigaud habitait Pétionville et était devenue une grande connaisseuse du vodou (cf. Alfred Métraux qui lui dédie du reste son ouvrage Le Vaudou haitien, Paris, Gallimard, 1958, notamment p. 13). Leiris, dans son carnet 2, à la date du 27 septembre 1948, la décrit comme « une grande femme brune opulente, avec parfois un air inspiré et autoritaire de papesse, parfois un éclatement de rire un peu canaille». Les archives d'Odette Mennesson-Rigaud concernant le vodou (carnets d'observation et photographies) ont été réunies et sont conservées à la Bibliothèque des Pères du Saint-Esprit de Port-au-Prince.

5. Cf. Alfred Métraux, Le Vaudou haitien, Paris, Gallimard, 1968,pp. 287-317 (2e édition reprise en collection « Tel », 1977).

6. Elle fait partie des archives ethnographiques de Michel Leiris, déposées au Laboratoire d'anthropologie sociale du Collège de France.

7. D'entrée de jeu, cette adresse rompt désagréablement avec l'usage qu'Odette MennessonRigaud avait institué dans la correspondance avec Leiris, l'appelant jusqu'alors " Mon cher Ami ». 8. Datée 13 févier 1952 et qui est jointe en effet à cette lettre du16 février. Outre la diatribe contre Leiris qu'elle conclut en ces termes : "Quand on quitte un pays on secoue la poussière de ses souliers sur ceux qui vous y ont reçu! », Odette Mennesson-Rigaud s'en prend directement à Métraux pour avoir en partie réécrit l'article qu'elle avait rédigé pour le numéro de Présence africaine (" Noël vodou en Haïti », pp. 37-60),y avoir fait des coupes et altéré ainsi sa pensée. Mais, dans une lettre à Leiris du 6 mars 1952 et d'après la réponse que Métraux lui aurait faite, on 
apprend que c'est à Georges Balandier, alors rédacteur en chef de Présence africaine, que Métraux avait confié la tâche de réviser et remanier son article. À noter, par ailleurs, que, selon une précédente lettre d'Odette Mennesson-Rigaud à Leiris (15 avril 1950), Claude Lévi-Strauss, qui allait publier moins de deux ans plus tard son beau texte sur le « Père Noël supplicié » (Les Temps Modernes, 1952, n 77, 1952), aurait été intéressé par l'ensemble des études de celle-ci sur le Noël vodou: "À propos de la publication de quelques-unes de mes études par Lévi-Strauss, nous en avons longuement parlé avec Métraux, avant son départ et nous étions plus ou moins tombés d'accord sur ce qui pourrait être publié. J'avais même commencé à grouper les éléments et tracé le plan de ce qui avait trait à la fête de Noël. Malheureusement le procès de mon mari a interrompu tout cela...».

9. Cf. Paul Reboux, Blancs et Noirs. Carnets de voyage, Haïti, Cuba, Paris, Flammarion, 1915.

10. Présence africaine a été fondée et dirigée par le Sénégalais Alioune Diop, mais André Gide, Albert Camus, Jean-Paul Sartre, Michel Leiris, Emmanuel Mounier, Pierre Naville et Paul Rivet comptaient parmi les membres du comité de patronage.

11. Période qui, comme nous le verrons plus bas, n'est pas sans signification. Il n'y a aucune raison de penser, étant donné l'état de conservation et de classement, presque maniaque, dans lequel Leiris tenait ses archives épistolaires, littéraires ou ethnographiques, que les lettres qu'il aurait reçues d'elle pendant cette période se fussent égarées.

12. En 1953, sorti de prison, il publiera un ouvrage « dominé par des préoccupations occultistes » (Alfred Métraux, Le Vaudou haïtien, op. cit., 1968, p. 16) sur La Tradition vaudou et le vaudou haïtien, Paris, Niclaus.

13. Dix-huit mois plus tôt Leiris avait publié son célèbre article sur «L'ethnographe devant le colonialisme » où il plaidait pour une « ethnologie engagée » (Les Temps Modernes, 1950, n 58, pp. 357-374, repris dans Brisées, Paris, Gallimard, 1992, pp. 141-164).

14. Haïti fut occupé par les États-Unis de 1915 à 1934.

15. «[...] et j'étais très fâchée aussi parce que je devais entendre de méchantes choses sur vous et que je ne pouvais pas vous défendre. Notez qu'en plusieurs fois il fut ajouté que cela étonnait de vous et que le souvenir que aviez laissé ne concordait pas avec ce que l'on avait à vous reprocher actuellement, mais qu'on "avait toujours tort de se fier à ceux qui montraient de l'intérêt pour Haïti” (vous connaissez l'antienne)» (lettre du 6 mars 1952).

16. Pour une approche théorique de la restitution en anthropologie, cf. l'article de Françoise Zonabend, «De l'objet et de sa restitution en anthropologie », Gradhiva, 1994, n 16, pp 3-14.

17. Cf. Odette Mennesson-Rigaud, "The Feasting of the Gods in Haitian Vodu ", Primitive Man, 1946, 19 (1-2), pp. 1-58.

18. Il est piquant d'observer que le premier texte que Leiris a publié au retour de la Mission Dakar-Djibouti concerne le sacrifice d'un taureau chez les adeptes des génies zar en Éthiopie; il s'agit d'un commentaire - extrait de son journal intime et des notes du principal informateur et interprète de Leiris, Abba Jérôme - de douze photographies prises pas ses compagnons : cf. Michel Leiris, « Le taureau de Seyfou Tchenger », Minotaure, 1933, n 3-4, pp. 75-84.

19. Cf. Jean Jamin, «Quand le sacré devint gauche », L'Ire des vents, 1981, n³-4, pp. 98-118.

20. Notion que Leiris avait puisée chez les concepteurs de la revue précisément intitulée Documents (1929-1930) dont, avec Georges Bataille, il fut l'un des principaux rédacteurs. Comme le note Denis Hollier, le document, tel que le conçoivent les animateurs de la revue, est censé restituer le réel en «fac-similé, non-métaphorisé, non-assimilé, non-idéalisé " ( La valeur d'usage de l'impossible ", préface à la réimpression de Documents, Paris, Jean-Michel Place, 1991, p. xx). Mais peut-il y avoir un fac-similé d'une relation comme l'est, .../.......... par exemple, la relation ethnographique ? D'une certaine manière et malgré son désir de comprendre les sociétés exotiques en termes de configuration politique et sociale, voire de rapports de force, Leiris ne parviendra jamais à se défaire d'une vision essentialiste de celles-ci, c'est-à-dire « de la croyance en l'existence de sociétés qui, à l'image des poètes, auraient décidé, par ce qui serait l'équivalent 
d'un choix originel à l'échelle de l'histoire de l'humanité, de tourner le dos aux valeurs technologiques » ou aux valeurs occidentales (cf. Denis Hollier, "Notice de Fourbis », in Michel Leiris, La Règle du jeu, Paris, Gallimard, 2003, p. 1404, « Bibliothèque de la Pléiade »). En ce sens le document, cueilli sur le vif, interviendrait comme une pièce à conviction de ce choix.

21. Cf. Jean Bazin, «L'anthropologie en question : altérité ou différence? ", in L'Université de tous les savoirs, L'Histoire, la sociologie et l'anthropologie, Paris, Odile Jacob, 2002, pp. 77-92.

22. Jean Bazin, « Interpréter ou décrire. Note critique sur la connaissance anthropologique », in Jacques Revel \& Nathan Wachtel, eds, Une École pour les sciences sociales, Paris, Cerf-Éditions de l'École des hautes études en sciences sociales, 1996, p. 412.

23. En 1950, Leiris est embarqué dans la rédaction du deuxième tome de La Règle du jeu : Fourbis.

24. Contact de civilisations en Martinique et en Guadeloupe, Paris, Gallimard-Unesco, 1955.

25. Cf. dans ce numéro l'article de Christine Laurière.

26. Il rédigera même une préface au moment de sa réédition en 1968 dans la «Bibliothèque des sciences humaines ", cinq ans après la disparition de Métraux. Mais il est vrai que, la même année, Leiris publie, en un temps record son étude sur les génies zar (il la rédige en trois mois) : La Possession et ses aspects théâtraux chez les Éthiopiens de Gondar, Paris, Plon, 1958 (repris dans Miroir de l'Afrique, op. cit., 1996).

27. Cf. Michel Leiris, «Titres et travaux ", Gradhiva, 1989, n 9, p. 8, repris dans C'est-à-dire, Paris, Éditions Jean-Michel Place, 1992.

28. Cela est notamment perceptible dans son article rédigé en 1935, « La croyance aux génies zar en Éthiopie du Nord », et publié en 1938 dans le Journal de psychologie normale et pathologique, 35 (1-2),pp. 107-125.

29. Dans notre édition de Miroir de l'Afrique, Jacques Mercier et moi avons insisté sur ces influences. Leiris lui-même, dans le prière d'insérer qu'il rédigea pour la réédition de La Possession et ses aspects théâtraux... en 1980 (composé en quatrième de couverture), reconnaît sa dette envers l'un d'entre eux: "L'importance que Michel Leiris", écrit-il, "accorde au théâtre et aux spectacles en général ne pouvait que le porter à étudier, avec le désir opiniâtre d'en discerner les dessous psychologiques, l'espèce de "comédie rituelle" (selon les termes d'Alfred Métraux) à laquelle s'adonnent les adeptes de cultes de possession comme celui des zar en Éthiopie et comme le vaudou haïtien. Au moins dans ces deux cas, il semble que les crises de possession tendent à constituer une sorte de théâtre vécu, mettant en jeu des personnages mythiques mais doués invisiblement d'une vie, et incarnés, plutôt que joués, par ceux ou celles qui les endossent temporairement. »

30. Dirigé par Georges Balandier et Jacques Maquet, Paris, Hazan, 1968, pp. 442-443.

31. «Présentation » de La Possession et ses aspects théâtraux..., in Miroir de l'Afrique, op. cit., 1996, p. 906.

32. Cf. supra note 18 .

\section{INDEX}

Mots-clés : enquête ethnographique, Haïti, observation, restitution, rite

Keywords : ethno-graphic survey, feed-back 


\section{AUTEUR}

\section{JEAN JAMIN}

École des hautes études en sciences sociales, laboratoire d'anthropologie et d'histoire de l'institution de la culture, jamin@ehess.fr 